\title{
TRIPLE- DIFFUSIVE CONVECTION IN A MICROPOLAR FERROFLUID IN THE PRESENCE OF ROTATION
}

\author{
S. CHAND \\ Department of Mathematics, SCVB Govt. College Palampur \\ HP-176061 INDIA \\ E-mail: suresh-9@hotmail.com
}

\begin{abstract}
This paper deals with the theoretical investigation of the triple-diffusive convection in a micropolar ferrofluid layer heated and soluted below subjected to a transverse uniform magnetic field in the presence of uniform vertical rotation. For a flat fluid layer contained between two free boundaries, an exact solution is obtained. A linear stability analysis theory and normal mode analysis method have been employed to study the onset convection. The influence of various parameters like rotation, solute gradients, and micropolar parameters (i.e., the coupling parameter, spin diffusion parameter and micropolar heat conduction parameter) on the onset of stationary convection has been analyzed. The critical magnetic thermal Rayleigh number for the onset of instability is also determined numerically for sufficiently large value of the buoyancy magnetization parameter $M_{l}$ (ratio of the magnetic to gravitational forces). The principle of exchange of stabilities is found to hold true for the micropolar fluid heated from below in the absence of micropolar viscous effect, microinertia, solute gradient and rotation. The oscillatory modes are introduced due to the presence of the micropolar viscous effect, microinertia, solute gradient and rotation, which were non-existent in their absence. In this paper, an attempt is also made to obtain the sufficient conditions for the non-existence of overstability.
\end{abstract}

Key words: triple- diffusive convection, micropolar ferrofluid, thermal convection, solute gradient, vertical magnetic field, rotation, magnetization.

\section{Introduction}

Micropolar fluids are fluids with internal structures in which coupling between the spin of each particle and the microscopic velocity field is taken into account. They represent fluids consisiting of rigid, randomly oriented or spherical particles suspended in a viscous medium, where the deformation of fluid particles is ignored (e.g., polymeric suspension, animal blood, liquid crystal). Micropolar fluids have received a great deal of research focus and interest due to their application in a number of processes that occur in industry. Such applications include the extrusion of polymer fluids, solidification of liquid crystal, cooling of metallic plate in a bath, exotic lubricants and colloidal suspension solutions. The micropolar fluid theory was introduced by Eringen (1966) in order to describe some physical systems, which do not satisfy the Navier-Stokes equation. The equations governing the micropolar fluid involve a spin vector and microinertia tensor in addition to the velocity vector. The theory can be used to explain the flow of colloidal fluids, liquid crystals, animal blood etc. The generalization of the theory including thermal effects was developed by Kazakia and Ariman (1971) and Eringen (1972). The theory of thermomicropolar convection began with Datta and Sastry (1976) and was interestingly continued by Ahmadi (1976), Lebon and PerezGarcia (1981), Bhattacharya and Jena (1983), Payne and Straughan (1989), Sharma and Kumar (1995; 1997) and Sharma and Gupta (1995). The above works give a good understanding of thermal convection in micropolar fluids.

In many situations involving suspensions, as in the magnetic fluid case, it might be pertinent to demand an Eringen micropolar description. This was suggested, in fact, by Rosenweig (1995) in his monograph. An interesting possibilities in a planer micropolar ferrofluid flow with an AC magnetic field was considered by Zahn and Greer (1995). They examined a simpler case where the applied magnetic fields 
along and transverse to the duct axis are spatially uniform and varying sinusoidally with time. In a uniform magnetic field, the magnetization characteristic depends on particle spin but does not depend on fluid velocity. Micropolar ferrofluid stabilities have become an important field of research these days. A particular stability problem is the Rayleigh-Bénard instability in a horizontal thin layer of a fluid heated from below. A detailed account of thermal convection in a horizontal thin layer of a Newtonian fluid heated from below was given by Chadrasekhar (1981). For a ferrofluid, a thermo-mechanical interaction is predicted by Finlayson (1970) in the presence of a uniform vertical magnetic field provided the magnetization is a function of temperature and magnetic field, and a temperature gradient is established across the fluid layer. The thermal convection in a Newtonian ferro fluid has been studied by many authors (Lalas and Carmi, 1971; Gotoh and Yamada, 1982; Stiles and Kagan, 1990; Siddeshwar, 1993; 1995; Sunil et al., 2004; 2005; 2005; 2005; 2006).

The Rayleigh-Bénard convection in a micropolar ferrofluid layer permeated by a uniform, vertical magnetic field with free-free, isothermal, spin-vanishing, magnetic boundaries was considered by Abraham (2002). She observed that the micropolar ferro fluid layer heated from below is more stable as compared with the classical Newtonian ferrofluid. The effect of rotation on thermal convection in micropolar fluids is important in certain chemical engineering and biochemical situations. Qin and Kaloni (1992) considered a thermal instability problem in a rotating micropolar fluid. They found that, depending upon the values of various micropolar parameters and the low values of the Taylor number, rotation has a stabilizing effect. The effect of rotation on thermal convection in micropolar fluids was also studied by Sharma and Kumar (1994), whereas the numerical solution of thermal instability of rotating micropolar fluid were discussed by Sastry and Rao (1983) without taking into account the rotation effect in the angular momentum equation. But we also appreciate the work of Bhattacharyya and Abbas (1985) and Qin and Kaloni (1992). They considered the effect of rotation in the angular momentum equation. More recently, Sunil et al. (2006), Sunil and Mahajan (2009), Sunil et al. (2008) studied the effect of rotation on the thermal convection problems in ferrofluids.

In the standard Bénard problem, the instability is driven by a density difference caused by a temperature difference between the upper and lower planes bounding the fluid. If the fluid, additionally has salt dissolved in it, then there are potentially two destabilizing sources for the density difference, the temperature field and salt field. The solution behavior in the double-diffusive convection problem is more interesting than that of the single component situation in so much as new instability phenomena may occur which are not present in the classical Bénard problem. When temperature and two or more component agents, or three different salts, are present, then the physical and mathematical situation becomes increasingly richer. Very interesting results in triply diffusive convection have been obtained by Pearlstein et al. (1989). The results of Pearlstein et al. (1989), are remarkable. They demonstrate that for triple diffusive convection linear instability can occur in discrete sections of the Rayleigh number domain with the fluid being linearly stable in a region inbetween the linear instability. This is because for certain parameters the neutral curve has a finite isolated oscillatory instability curve lying below the usual unbounded stationary convection. Straughan and Walker (1997) derive the equations for non-Boussinesq convection in a multicomponent fluid and investigate the situation analogous to that of Pearlstein et al. (1989), but allowing for a density non linearity in the temperature field. Lopez et al. (1990) derive an equivalent problem with fixed boundary conditions and show that the effect of the boundary conditions breaks the perfect symmetry. In reality the density of a fluid is never a linear function of temperature, and so the work of Straughan and Walker applies to the general situation where the equation of state is one of the density quadratic in temperature. This is important, since they find that a departure from the linear Boussinesq equation of state changes the perfect symmetry of the heart shaped neutral curve of Pearlstein et al. (1989).

In view of the recent increase in the number of non iso-thermal situations wherein magnetic fluids are put to use in place of classical fluids, we intend to extend our work to the problem of thermal convection in Eringen's micropolar fluid to the triple-diffusive convection in a mocropolar ferrofluid in the presence of rotation. In the present analysis, for mathematical simplicity, we have not considered the effect of rotation in the angular momentum equation. 


\section{Mathematical formulation of the problem}

Here we consider an infinite, horizontal layer of thickness $d$ of an electrically non-conducting incompressible thin micropolar ferromagnetic fluid heated and salted from below. The temperature $T$ and solute concentrations $C^{1}$ and $C^{2}$ at the bottom and top surfaces $z=\mp \frac{1}{2} d$ are $T_{0}$ and $T_{1} ; C_{0}{ }^{1}$ and $C_{1}{ }^{1}$; and $C_{0}{ }^{2}$ and $C_{1}^{2}$ respectively, and a uniform temperature gradient $\beta\left(=\left|\frac{d T}{d Z}\right|\right)$ and uniform solute gradients are $\beta^{\prime}\left(=\left|\frac{d C^{I}}{d Z}\right|\right)$ and $\beta^{\prime \prime}\left(=\left|\frac{d C^{2}}{d Z}\right|\right)$ are maintained. Both the boundaries are taken to be free and perfect conductors of heat. The fluid layer is assumed to occupy the layer $z \in(-d / 2, d / 2)$ with gravity acting in the negative $z$ - direction and magnetic field, $\boldsymbol{H}=H_{0}{ }^{\mathrm{ext}} \widehat{\boldsymbol{k}}$, where $\widehat{\boldsymbol{k}}=(0,0,1)$, acts outside the layer. The whole system is assumed to rotate with angular velocity $\boldsymbol{\Omega}=(0,0, \Omega)$ along the vertical axis, which is taken as the $z$-axis.

The mathematical equations governing the motion of incompressible micropolar ferrofluids (utilizing Boussinesq approximation) for the above model are as follows:

The continuity equation for an incompressible fluid is

$$
\nabla \cdot \boldsymbol{q}=0
$$

The momentum and internal angular momentum equations are

$$
\begin{aligned}
& \rho_{0}\left[\frac{\partial}{\partial t}+(\boldsymbol{q} \cdot \nabla)\right] \boldsymbol{q}=-\nabla p+\rho \boldsymbol{g}+\mu_{0}(\boldsymbol{M} \cdot \nabla) \boldsymbol{H}+(\varsigma+\eta) \nabla^{2} \boldsymbol{q}+2 \varsigma(\nabla \times \boldsymbol{\omega})+2 \rho_{0}(\boldsymbol{q} \times \boldsymbol{\Omega}), \\
& \rho_{0} I\left[\frac{\partial}{\partial t}+(\boldsymbol{q} \cdot \nabla)\right] \boldsymbol{\omega}=2 \varsigma(\nabla \times \boldsymbol{q}-2 \boldsymbol{\omega})+\mu_{0}(\boldsymbol{M} \times \boldsymbol{H})+\left(\lambda^{\prime}+\eta^{\prime}\right) \nabla(\nabla \cdot \boldsymbol{\omega})+\eta^{\prime} \nabla^{2} \boldsymbol{\omega} .
\end{aligned}
$$

The temperature and solute concentration equations for an incompressible micropolar ferromagnetic fluid are

$$
\begin{aligned}
& {\left[\rho_{0} C_{V, H}-\mu_{0} \boldsymbol{H}\left(\frac{\partial \boldsymbol{M}}{\partial t}\right)_{V, H}\right] \frac{D T}{D t}+\mu_{0} T\left(\frac{\partial \boldsymbol{M}}{\partial t}\right)_{V, H} \cdot \frac{D H}{D t}=K_{l} \nabla^{2} T+\delta(\nabla \times \boldsymbol{\omega}) \cdot \nabla T,} \\
& {\left[\rho_{0} C_{V, H}-\mu_{0} \boldsymbol{H}\left(\frac{\partial \boldsymbol{M}}{\partial C^{l}}\right)_{V, H}\right] \frac{D C^{l}}{D t}+\mu_{0}\left(C^{l} \frac{\partial \boldsymbol{M}}{\partial C^{l}}\right)_{V, H} \cdot \frac{D H}{D t}=K_{l}^{\prime} \nabla^{2} C^{l},} \\
& {\left[\rho_{0} C_{V, H}-\mu_{0} \boldsymbol{H}\left(\frac{\partial \boldsymbol{M}}{\partial C^{2}}\right)_{V, H}\right] \frac{D C^{2}}{D t}+\mu_{0} C^{2}\left(\frac{\partial \boldsymbol{M}}{\partial C^{2}}\right)_{V, H} \cdot \frac{D H}{D t}=K_{l}^{\prime \prime} \nabla^{2} C^{2} .}
\end{aligned}
$$

In terms of temperature $T$ and the concentrations $C^{1}$ and $C^{2}$, we suppose the density of the mixture is given by (known as the density equation of state)

$$
\rho=\rho_{0}\left[1-\alpha\left(T-T_{a}\right)+\alpha^{\prime}\left(C^{1}-C_{a}{ }^{l}\right)+\alpha^{\prime \prime}\left(C^{2}-C_{a}{ }^{2}\right]\right.
$$


where $\rho, \rho_{0}, \boldsymbol{q}, \boldsymbol{\omega}, t, p, \eta, \varsigma, \lambda^{\prime}, \eta^{\prime}, \delta, I, \mu_{0}, \boldsymbol{B}, C_{V, H}, M, K_{1}, K_{l}^{\prime}, K_{l}^{\prime \prime}, \alpha, \alpha^{\prime}, \alpha^{\prime \prime}$ are the fluid density, reference density, velocity, microrotation, time, pressure, shear kinematic viscosity coefficient, coupling viscosity coefficient or vortex viscosity, bulk spin viscosity coefficient, shear spin viscosity coefficient, micropolar heat conduction coefficient, moment of inertia (microinertia constant),magnetic permeability, magnetic induction, heat capacity at constant volume and magnetic field, magnetization, thermal conductivity, solute conductivity, thermal expansion coefficient and concentration expansion coefficient analogous to the thermal expansion coefficient, respectively. $T_{a}$ is the average temperature given by $T_{a}=\left(T_{0}+T_{1}\right) / 2$ where $T_{0}$ and $T_{l}$ are the constant average temperatures of the lower and upper surfaces of the layer and $C_{a}{ }^{1}$ and $C_{a}{ }^{2}$ are the average concentrations given by $C_{a}{ }^{l}=\left(C_{0}{ }^{1}+C_{1}{ }^{1}\right) / 2$ and $C_{a}{ }^{2}=\left(C_{0}{ }^{2}+C_{1}{ }^{2}\right) / 2$, where $C_{0}{ }^{1}, C_{1}{ }^{l}$ and $C_{0}{ }^{2}, C_{1}{ }^{2}$ are the constant average concentrations of the lower and upper surfaces of the layer. The partial derivatives of $\boldsymbol{M}$ are the material properties that can be evaluated once the magnetic equation of state, such as Eq.(2.10) below is known. In writing Eq.(2.2), we also use the Boussinesq approximation by allowing the density to change only in the gravitational body force term.

Maxwell's equation, simplified for a non-conducting fluid with no displacement currents, becomes

$$
\begin{aligned}
& \nabla \cdot \boldsymbol{B}=0, \\
& \nabla \times \boldsymbol{H}=0
\end{aligned}
$$

where the magnetic induction is given by

$$
\boldsymbol{B}=\mu_{0}(\boldsymbol{H}+\boldsymbol{M}) .
$$

We assume that the magnetization is aligned with the magnetic field, but allow a dependence on the magnitude of the magnetic field, temperature and salinity, so that

$$
\boldsymbol{M}=\frac{\boldsymbol{H}}{H} M\left(H, T, C^{1}, C^{2}\right) .
$$

The magnetic equation of state is linearized about the magnetic field, $H_{0}$, an average temperature, $T_{a}$, and average concentrations, $C_{a}{ }^{1}$ and $C_{a}{ }^{2}$ to become

$$
M=M_{0}+\chi\left(H-H_{0}\right)-K_{2}\left(T-T_{a}\right)+K_{3}\left(C^{1}-C_{a}{ }^{l}\right)+K_{4}\left(C^{2}-C_{a}{ }^{2}\right)
$$

where magnetic susceptibility, pyromagnetic coefficient and salinity magnetic coefficients are defined by

$$
\begin{aligned}
& \chi \equiv\left(\frac{\partial M}{\partial H}\right) H_{0}, T_{a}, \quad K_{2} \equiv-\left(\frac{\partial M}{\partial T}\right) H_{0}, T_{a}, \\
& K_{3} \equiv\left(\frac{\partial M}{\partial C^{1}}\right) H_{0}, C_{a}^{1} \text { and } K_{4} \equiv\left(\frac{\partial M}{\partial C^{2}}\right) H_{0}, C_{a}^{2}, \quad \text { respectively. }
\end{aligned}
$$

Here $H_{0}$ is the uniform magnetic field of the fluid layer when placed in an external magnetic field $H=H_{0}{ }^{\text {ext }} \hat{k}$, where $\hat{k}$ is a unit vector in the $z$ direction

$$
H=|\boldsymbol{H}|, \quad M=|\boldsymbol{M}| \quad \text { and } \quad M_{0}=M\left(H_{0}, T_{a}, C_{a}{ }^{l}, C_{a}{ }^{2}\right) .
$$


The effect of rotation contributes two terms: (a) centrifugal force $-\left(\rho_{0} / 2\right) \operatorname{grad}|\boldsymbol{\Omega} \times r|^{2}$ and (b) Coriolis force $2 \rho_{0}(\boldsymbol{q} \times \boldsymbol{\Omega})$. In Eq.(2.2), $p=p_{f}-\frac{1}{2} \rho_{0}|\boldsymbol{\Omega} \times r|^{2}$ is the reduced pressure, where $p_{f}$ stands for the fluid pressure.

The basic state is assumed to be a quiescent state and is given by

$$
\begin{aligned}
& \boldsymbol{q}=\boldsymbol{q}_{\boldsymbol{b}}=(0,0,0), \quad \boldsymbol{\omega}=\boldsymbol{\omega}_{\boldsymbol{b}}=(0,0,0), \quad \rho=\rho_{b}(z), \quad p=p_{b}(z), \quad T=T_{b}(z)=-\beta z+T_{a}, \\
& C^{1}=C_{b}^{1}(z)=-\beta z+C_{a}^{1}, \quad C^{2}=C_{b}^{2}(z)=-\beta z+C_{a}^{2}, \quad \beta=\left(T_{0}-T_{1}\right) / d, \\
& \beta^{\prime}=\left(C_{1}^{1}-C_{0}^{1}\right) / d, \quad \beta^{\prime \prime}=\left(C_{1}^{2}-C_{0}^{2}\right) / d, \\
& \boldsymbol{H}_{\boldsymbol{b}}=\left[H_{0}-\frac{K_{2} \beta z}{1+\chi}+\frac{K_{3} \beta^{\prime} z}{1+\chi}+\frac{K_{4} \beta^{\prime \prime} z}{1+\chi}\right] \hat{\boldsymbol{k}}, \quad \boldsymbol{M}_{\boldsymbol{b}}=\left[M_{0}+\frac{K_{2} \beta z}{1+\chi}+\frac{K_{3} \beta^{\prime} z}{1+\chi}+\frac{K_{4} \beta^{\prime \prime} z}{1+\chi}\right] \hat{\boldsymbol{k}}, \\
& \text { and } \quad H_{0}+M_{0}=H_{0}^{e x t}
\end{aligned}
$$

where the subscript ${ }^{\prime} b$ ' denotes the basic state.

\section{The perturbation equations and normal mode analysis method}

We now examine the stability of the basic state, and assume that the perturbation quantities are small. We write

$$
\begin{aligned}
& \boldsymbol{q}=\boldsymbol{q}_{\boldsymbol{b}}+\boldsymbol{q}^{\prime}, \quad \boldsymbol{\omega}=\boldsymbol{\omega}_{\boldsymbol{b}}+\boldsymbol{\omega}^{\prime}, \quad \rho=\rho_{b}+\rho^{\prime}, \quad p=p_{b}(z)+p^{\prime}, \quad T=T_{b}(z)+\theta, \\
& C^{l}=C^{l}{ }_{b}(z)+\gamma, \quad C^{2}=C^{2}{ }_{b}(z)+\gamma^{\prime}, \quad H=H_{b}(z)+H^{\prime} \quad \text { and } \quad M=M_{b}(z)+M^{\prime}
\end{aligned}
$$

where $\boldsymbol{q}^{\prime}=(u, v, w), \boldsymbol{\omega}^{\prime}=\left(\omega_{1}, \omega_{2}, \omega_{3}\right), \rho^{\prime}, \theta, \gamma, \gamma^{\prime}, \boldsymbol{H}^{\prime}, \boldsymbol{M}^{\prime}$ are perturbations in velocity $\boldsymbol{q}$, spin $\boldsymbol{\omega}$, pressure $p$, temperature $T$, concentrations $C^{l}$ and $C^{2}$, magnetic field intensity $\boldsymbol{H}$, and magnetization $\boldsymbol{M}$, respectively. The change in density $\rho^{\prime}$, caused mainly by the perturbations $\theta, \gamma$, and $\gamma^{\prime}$ in temperature and concentrations, respectively, is given by

$$
\rho^{\prime}=-\rho_{0}\left(\alpha \theta-\alpha^{\prime} \gamma-\alpha^{\prime \prime} \gamma^{\prime}\right)
$$

Then, the linearized perturbation equations (by neglecting second-order small quantities) of the micropolar ferromagnetic fluid become

$$
\begin{aligned}
& \rho_{0} \frac{\partial u}{\partial t}=-\frac{\partial p^{\prime}}{\partial x}+\mu_{0}\left(M_{0}+H_{0}\right) \frac{\partial H_{1}^{\prime}}{\partial z}+(\varsigma+\eta) \nabla^{2} u+2 \varsigma \Omega_{l}^{\prime}+2 \rho_{0} \Omega_{V}, \\
& \rho_{0} \frac{\partial v}{\partial t}=-\frac{\partial p^{\prime}}{\partial y}+\mu_{0}\left(M_{0}+H_{0}\right) \frac{\partial H_{2}^{\prime}}{\partial z}+(\varsigma+\eta) \nabla^{2} v+2 \varsigma \Omega_{2}^{\prime}-2 \rho_{0} \Omega_{u},
\end{aligned}
$$




$$
\begin{aligned}
& \rho_{0} \frac{\partial w}{\partial t}=-\frac{\partial p^{\prime}}{\partial z}+\mu_{0}\left(M_{0}+H_{0}\right) \frac{\partial H_{3}^{\prime}}{\partial z}+(\varsigma+\eta) \nabla^{2} w+2 \varsigma \Omega_{3}-\mu_{0} \frac{K_{2} \beta}{1+\chi}\left\{H_{3}^{\prime}(1+\chi)+K_{2} \theta\right\}+ \\
& +\mu_{0} \frac{K_{3} \beta^{\prime}}{1+\chi}\left\{H_{3}^{\prime}(1+\chi)+K_{3} \gamma\right\}+\mu_{0} \frac{K_{4} \beta^{\prime \prime}}{1+\chi}\left\{H_{3}^{\prime}(1+\chi)+K_{4} \gamma^{\prime}\right\}-\mu_{0} \frac{K_{2} K_{3}}{1+\chi}\left(\beta^{\prime} \theta+\beta \gamma\right)+ \\
& +\mu_{0} \frac{K_{3} K_{4}}{1+\chi}\left(\beta^{\prime \prime} \gamma+\beta \gamma^{\prime}\right)-\mu_{0} \frac{K_{2} K_{4}}{1+\chi}\left(\beta^{\prime \prime} \theta+\beta \gamma\right)+\rho_{0} g\left(\alpha \theta-\alpha^{\prime} \gamma-\alpha^{\prime \prime} \gamma^{\prime}\right) \\
& \rho_{0}^{I} \frac{\partial \omega^{\prime}}{\partial t}=2 \varsigma\left(\nabla \times \boldsymbol{q}^{\prime}-2 \omega^{\prime}\right)+\mu_{0}\left(\boldsymbol{M}_{\boldsymbol{b}} \times \boldsymbol{H}^{\prime}+\boldsymbol{M}^{\prime} \times \boldsymbol{H}_{\boldsymbol{b}}\right)+\left(\lambda^{\prime}+\eta^{\prime}\right) \nabla\left(\nabla \cdot \omega^{\prime}\right)+\eta^{\prime} \nabla^{2} \omega^{\prime} \\
& \frac{\partial u}{\partial x}+\frac{\partial v}{\partial y}+\frac{\partial w}{\partial z}=0, \\
& \rho C_{1} \frac{\partial \theta}{\partial t}-\mu_{0} T_{0} K_{2} \frac{\partial}{\partial t}\left(\frac{\partial \phi 1^{\prime}}{\partial z}\right)=K_{1} \nabla^{2} \theta+\left[\rho C_{1} \beta-\mu_{0} T_{0} K_{2}^{2} \beta /(1+\chi)\right] \omega-\delta \beta \Omega_{3}^{\prime} \\
& \rho C_{1}^{\prime} \frac{\partial \gamma}{\partial t}-\mu_{0} C_{0}^{l} K_{3} \frac{\partial}{\partial t}\left(\frac{\partial \phi 2^{\prime}}{\partial z}\right)=K_{l}^{\prime} \nabla^{2} \gamma+\left[\rho C_{l}^{\prime} \beta^{\prime}-\mu_{0} C_{0}^{l} K_{3}^{2} \beta^{\prime} /(1+\chi)\right] \omega \\
& \rho C_{1}^{\prime \prime} \frac{\partial \gamma^{\prime}}{\partial t}-\mu_{0} C_{0}^{2} K_{2} \frac{\partial}{\partial t}\left(\frac{\partial \phi 3^{\prime}}{\partial z}\right)=K_{l}^{\prime \prime} \nabla^{2} \gamma^{\prime}+\left[\rho C_{l}^{\prime \prime} \beta^{\prime \prime}-\mu_{0} C_{0}^{2} K_{2}^{2} \beta^{\prime \prime} /(1+\chi)\right] \omega
\end{aligned}
$$

where $\rho C_{l}=\rho_{0} C_{V, H}+\mu_{0} K_{2} \boldsymbol{H}_{0} ; \rho C_{l}{ }^{\prime}=\rho_{0} C_{V, H}-\mu_{0} K_{3} \boldsymbol{H}_{0} ; \rho C_{l}{ }^{\prime \prime}=\rho_{0} C_{V, H}-\mu_{0} K_{4} \boldsymbol{H}_{\boldsymbol{0}}$.

Equation (2.9) and Eq.(2.10) yield

$$
\begin{aligned}
& H_{3}^{\prime}+M_{3}^{\prime}=(1+\chi) H_{3}^{\prime}-K_{2} \theta, \\
& H_{3}^{\prime}+M_{3}^{\prime}=(1+\chi) H_{3}^{\prime}-K_{3} \gamma, \\
& H_{3}^{\prime}+M_{3}^{\prime}=(1+\chi) H_{3}^{\prime}-K_{4} \gamma^{\prime}, \\
& H_{i}^{\prime}+M_{i}^{\prime}=\left(1+M_{0} / H_{0}\right) H_{i}^{\prime} \quad i=1,2,3
\end{aligned}
$$

where, we have assumed $K_{2}\left(T_{b}-T_{a}\right)<<(1+\zeta) H_{0} ; K_{3} \beta^{\prime} d<<(1+\zeta) H_{0} ; K_{4} \beta^{\prime \prime} d<<(1+\zeta) H_{0} ; \Omega^{\prime}=\left(\Omega_{l}^{\prime}\right.$, $\left.\Omega_{2}{ }^{\prime}, \Omega_{3}{ }^{\prime}\right)=\left(\nabla \times \omega^{\prime}\right)$.

Thus the analysis is restricted to a physical situation in which the magnetization induced by temperature and concentration variations is small compared to that induced by the external magnetic field. Equation (2.7b) means that we can write $H^{\prime}=\nabla\left(\phi_{1}^{\prime}-\phi_{2}^{\prime}-\phi_{3}\right)$, where $\phi_{1}{ }^{\prime}$ is the perturbed magnetic potential and $\phi_{2}{ }^{\prime}, \phi_{3}{ }^{\prime}$ are the perturbed magnetic potentials analogous to the solute.

Eliminating $u, v, p^{\prime}$ between Eqs (3.3)-(3.5), using Eq.(3.6), and taking curl once on Eq.(2.3) and considering only $k^{\text {th }}$ component, we obtain 


$$
\begin{aligned}
& \left(\rho_{0} \frac{\partial}{\partial t}-(\varsigma+\eta) \nabla^{2}\right)=-\mu_{0} \frac{K_{2} \beta}{1+\chi} \nabla_{I}^{2}\left\{(1+\varsigma) \frac{\partial}{\partial z}\left(\phi_{1}^{\prime}-\phi_{2}^{\prime}-\phi_{3}^{\prime}\right)-K_{2} \theta\right\}+ \\
& +\mu_{0} \frac{K_{3} \beta^{\prime}}{1+\chi} \nabla_{1}^{2}\left\{(1+\varsigma) \frac{\partial}{\partial z}\left(\phi_{1}^{\prime}-\phi_{2}^{\prime}-\phi_{3}^{\prime}\right)-K_{3} \gamma\right\}+ \\
& +\mu_{0} \frac{K_{4} \beta^{\prime \prime}}{1+\chi} \nabla_{I}^{2}\left\{(1+\varsigma) \frac{\partial}{\partial z}\left(\phi_{1}^{\prime}-\phi_{2}^{\prime}-\phi_{3}^{\prime}\right)-K_{4} \gamma^{\prime}\right\}+ \\
& -\mu_{0} \frac{K_{2} K_{3}}{1+\chi} \nabla_{1}^{2}\left(\beta^{\prime} \theta+\beta \gamma\right)+\mu_{0} \frac{K_{4} K_{3}}{1+\chi} \nabla_{I}^{2}\left(\beta^{\prime \prime} \gamma+\beta^{\prime} \gamma^{\prime}\right)+ \\
& -\mu_{0} \frac{K_{2} K_{4}}{1+\chi} \nabla_{1}^{2}\left(\beta^{\prime \prime} \theta+\beta^{\prime} \gamma\right)+\rho_{0} g \nabla_{I}^{2}\left(\alpha \theta-\alpha^{\prime} \gamma-\alpha^{\prime \prime} \gamma^{\prime}\right)+2 \varsigma \nabla^{2} \Omega_{3}^{\prime}-2 \rho_{0} \Omega \frac{\partial \varsigma_{1}}{\partial Z}, \\
& \rho_{0} \mid \frac{\partial \Omega_{3}^{\prime}}{\partial t}=-2 \varsigma\left(\nabla^{2} \omega+2 \Omega_{3}^{\prime}\right)+\eta^{\prime} \nabla^{2} \Omega_{3}^{\prime} .
\end{aligned}
$$

The vertical component of the vorticity equation is

$$
\rho_{0} \frac{\partial \varsigma_{1}}{\partial t}=2 \rho_{0} \Omega \frac{\partial w}{\partial z}+(\varsigma+\eta) \nabla^{2} \varsigma_{1}
$$

where $\varsigma_{I}=\frac{\partial v}{\partial x}-\frac{\partial u}{\partial y}$ stands for the $z$-component of the vorticity.

From Eq.(3.8), we have

$$
\begin{aligned}
& (1+\chi) \frac{\partial^{2} \phi 1^{\prime}}{\partial z^{2}}+\left(1+\frac{M_{0}}{H_{0}}\right) \nabla^{2} \phi_{1}^{\prime}-K_{2} \frac{\partial \theta}{\partial Z}=0, \\
& (1+\chi) \frac{\partial^{2} \phi 2^{\prime}}{\partial z^{2}}+\left(1+\frac{M_{0}}{H_{0}}\right) \nabla^{2} \phi_{2}^{\prime}-K_{3} \frac{\partial \gamma}{\partial Z}=0, \\
& (1+\chi) \frac{\partial^{2} \phi 3^{\prime}}{\partial z^{2}}+\left(1+\frac{M_{0}}{H_{0}}\right) \nabla^{2} \phi_{3}^{\prime}-K_{4} \frac{\partial \gamma^{\prime}}{\partial Z}=0 .
\end{aligned}
$$

We analyze the normal mode technique. This can be written

$$
f(x, y, z, t)=f(z, t) \exp i\left(k_{x} x+k_{y} y\right)
$$

where $f(z, t)$ represents $W(z, t), \Theta(z, t), Z(z, t), \Gamma(z, t), \Psi(z, t), \phi_{1}(z, t), \phi_{2}(z, t), \phi_{3}(z, t), \Omega_{3}(z, t) ; k_{x}, k_{y}$ are the wave numbers along the $x$ - and $y$-directions, respectively and $k=\sqrt{ }\left(k_{x}^{2}+k_{y}^{2}\right)$ is the resultant wave number.

Following the normal mode analysis, the linearized perturbation dimensionless equations are 


$$
\begin{aligned}
& \left\{\frac{\partial}{\partial t^{*}}-\left(1+N_{1}\right)\left(D^{2}-a^{2}\right)\right\}\left(D^{2}-a^{2}\right) W=a \sqrt{R}\left[\left(M_{1}-M_{4}\right) D \phi_{1}^{*}-\left(1+M_{1}-M_{4}\right) T^{*}\right]+ \\
& +a \sqrt{S_{1}}\left[\left(M_{1}^{\prime}-M_{4}^{\prime}\right) D \phi_{2}{ }^{*}-\left(1+M_{1}^{\prime}+M_{4}^{\prime}\right) C^{1 *}\right]+a \sqrt{S_{2}}\left[\left(M_{1}^{\prime \prime}-M_{4}^{\prime \prime}\right) D \phi_{3}{ }^{*}+\right. \\
& \left.+\left(1-M_{1}^{\prime \prime}+M_{4}^{\prime \prime}\right) C^{2 *}\right]+2 N_{1}\left(D^{2}-a^{2}\right) \Omega_{3}{ }^{*}-\sqrt{T_{A}} D Z^{*} \\
& \left\{\frac{\partial}{\partial t^{*}}-\left(1+N_{1}\right)\left(D^{2}-a^{2}\right)\right\} Z^{*}=\sqrt{T_{A}} D W^{*} \\
& I^{\prime} \frac{\partial \Omega_{3}{ }^{*}}{\partial t^{*}}=-2 N_{1}\left\{\left(D^{2}-a^{2}\right) W^{*}+2 \Omega_{3}^{*}\right\}+N_{3}\left(D^{2}-a^{2}\right) \Omega_{3}{ }^{*} \\
& \mathrm{P}_{\mathrm{r}} \frac{\partial T^{*}}{\partial t^{*}}-P_{r} M_{2} \frac{\partial}{\partial t^{*}}\left(D \phi_{1}^{*}\right)=\left(D^{2}-a^{2}\right) T^{*}+a \sqrt{R}\left(1-M_{2}\right) W^{*}-a \sqrt{R} N_{5} \Omega_{3}^{*} \\
& P s_{1} \frac{\partial C^{1 *}}{\partial t^{*}}-P s_{1} M_{2}^{\prime} \frac{\partial}{\partial t^{*}}\left(D \phi_{2}^{*}\right)=\left(D^{2}-a^{2}\right) C^{1 *}+a \sqrt{S_{1}}\left(1-M_{2}^{\prime}\right) W^{*} \\
& P s_{2} \frac{\partial C^{2 *}}{\partial t^{*}}-P s_{2} M_{2}^{\prime \prime} \frac{\partial}{\partial t^{*}}\left(D \phi_{3}^{*}\right)=\left(D^{2}-a^{2}\right) C^{2 *}+a \sqrt{S_{2}}\left(1-M_{2}^{\prime \prime}\right) W^{*} \\
& D^{2} \phi_{1}{ }^{*}-a^{2} M_{3} \phi_{1}{ }^{*}-D T^{*}=0 \\
& D^{2} \phi_{2}{ }^{*}-a^{2} M_{3} \phi_{2}{ }^{*}-D C^{1 *}=0 \\
& D^{2} \phi_{3}{ }^{*}-a^{2} M_{3} \phi_{3}{ }^{*}-D C^{2 *}=0
\end{aligned}
$$

where the following non dimension quantities and non dimensionless parameters are introduced

$$
\begin{aligned}
& t^{\prime}=\frac{v t}{d^{2}}, \quad W^{*}=\frac{W d}{v}, \quad \phi_{1}^{*}=\frac{(1+\chi) K_{1} a \sqrt{R}}{K_{2} \rho C_{1} \beta v d^{2}} \phi_{1}, \quad \phi_{2}^{*}=\frac{(1+\chi) K_{1}^{\prime} a \sqrt{S_{1}}}{K_{3} \rho C_{1}^{\prime} \beta^{\prime} v d^{2}} \phi_{2}, \quad \phi_{3}^{*}=\frac{(1+\chi) K_{1}^{\prime \prime} a \sqrt{S_{2}}}{K_{4} \rho C_{l}^{\prime \prime} \beta^{\prime \prime} v d^{2}} \phi_{3}, \\
& R_{1}=\frac{g \alpha \beta d^{4} \rho C_{1}}{v K_{1}}, \quad S_{1}=\frac{g \alpha^{\prime} \beta^{\prime} d^{4} \rho C_{1}^{\prime}}{v K_{1}^{\prime}}, \quad S_{2}=\frac{g \alpha^{\prime \prime} \beta^{\prime \prime} d^{4} \rho C_{1}^{\prime \prime}}{v K_{l}^{\prime \prime}}, \quad T^{*}=\frac{K_{1} a \sqrt{R}}{\rho C_{1} \beta v d} \Theta, \quad C^{1 *}=\frac{K_{1}^{\prime} a \sqrt{S_{1}}}{\rho C_{l}^{\prime} \beta^{\prime} v d} \Gamma, \\
& C^{2 *}=\frac{K_{l}^{\prime \prime} a \sqrt{S_{2}}}{\rho C_{l}^{\prime \prime} \beta^{\prime \prime} v d} \Psi, \quad a=k d, \quad z^{\prime}=\frac{z}{d}, \quad D=\frac{\partial}{\partial Z^{*}}, \quad \mathrm{P}_{\mathrm{r}}=\frac{v}{K_{1}} \rho C_{1}, \quad P_{s_{1}}=\frac{v}{K_{1}^{\prime}} \rho C_{1}^{\prime}, \\
& P_{s_{2}}=\frac{v}{K_{l}^{\prime \prime}} \rho C_{1}^{\prime \prime}, \quad T_{A}=\left(\frac{2 \Omega d^{2}}{v}\right)^{2}, \quad M_{1}=\frac{\mu_{0} K_{2}^{2} \beta}{(1+\chi) \alpha \rho_{0} g}, \quad M_{1}^{\prime}=\frac{\mu_{0} K_{3}^{2} \beta^{\prime}}{(1+\chi) \alpha^{\prime} \rho_{0} g}, \quad M_{1}^{\prime \prime}=\frac{\mu_{0} K_{4}^{2} \beta^{\prime \prime}}{(1+\chi) \alpha^{\prime \prime} \rho_{0} g},
\end{aligned}
$$




$$
\begin{aligned}
& M_{2}=\frac{\mu_{0} T_{0} K_{2}^{2}}{(1+\chi) \rho C_{1}}, \quad M_{2}^{\prime}=\frac{\mu_{0} C_{0}^{1} K_{3}^{2}}{(1+\chi) \rho C_{1}}, \quad M_{2}^{\prime \prime}=\frac{\mu_{0} C_{0}^{2} K_{4}^{2}}{(1+\chi) \rho C_{1}^{\prime \prime}}, \quad M_{3}=\frac{\left(1+\frac{M_{0}}{H_{0}}\right)}{(1+\chi)}, \quad M_{4}=\frac{\mu_{0} K_{2} K_{3} \beta^{\prime}}{(1+\chi) \alpha \rho_{0} g}, \\
& M_{4}^{\prime}=\frac{\mu_{0} K_{3} K_{4} \beta^{\prime \prime}}{(1+\chi) \alpha^{\prime} \rho_{0} g}, \quad M_{4}^{\prime \prime}=\frac{\mu_{0} K_{2} K_{4} \beta}{(1+\chi) \alpha^{\prime \prime} \rho_{0} g}, \quad M_{5}=\frac{M_{4}}{M_{1}}=\frac{M_{1}^{\prime}}{M_{4}^{\prime}}=\frac{K_{3} \beta^{\prime}}{K_{2} \beta}=\frac{M_{1}^{\prime \prime}}{M_{4}^{\prime \prime}}=\frac{K_{4} \beta^{\prime \prime}}{K_{2} \beta}, \quad N_{1}=\frac{\varsigma}{\eta} \\
& N_{3}=\frac{\eta^{\prime}}{\eta d^{2}}, \quad N_{5}=\frac{\delta}{\rho C_{1} d^{2}}, \quad I^{\prime}=\frac{I}{d^{2}} \quad \text { and } \quad \Omega_{3}^{*}=\frac{\Omega_{3} d^{3}}{v} .
\end{aligned}
$$

\section{Exact solution for free boundaries}

Here the simplest boundary conditions chosen, namely free-free, no- spin, isothermal with infinite magnetic susceptibility $\chi$ in the perturbed field keep the problem analytically tractable and serve the purpose of providing a qualitative insight into the problem. The case of two free boundaries is of little physical interest, but it is mathematically important because one can derive an exact solution, whose properties guide our analysis. Thus the exact solution of Eqs (3.22)-(3.30) subject to the boundary conditions are

$$
W^{*}=D 2 W=T^{*}=C^{1 *}=C^{2 *}=\Omega 3^{*}=D \phi 1^{*}=D \phi 2^{*}=D \phi 3^{*}=0 \quad \text { at } \quad z= \pm \frac{1}{2},
$$

is written in the form

$$
\begin{aligned}
& W^{*}=A_{1} e^{\sigma t^{*}} \cos \pi z^{*}, \quad T^{*}=B_{1} e^{\sigma t^{*}} \cos \pi z^{*}, \quad D \phi_{1}{ }^{*}=C_{1} e^{\sigma t^{*}} \cos \pi z^{*}, \quad \Omega_{3}{ }^{*}=D_{1} e^{\sigma t^{*}} \cos \pi z^{*} \\
& D \phi_{2}{ }^{*}=E_{1} e^{\sigma t^{*}} \cos \pi z^{*}, \quad \phi_{1}{ }^{*}=\left(\frac{C_{1}}{\pi}\right) e^{\sigma t^{*}} \sin \pi z^{*}, \quad \phi_{2}{ }^{*}=\left(\frac{E_{1}}{\pi}\right) e^{\sigma t^{*}} \sin \pi z^{*}, \quad C^{*}=F_{1} e^{\sigma t^{*}} \cos \pi z^{*} \\
& C^{2 *}=G_{1} e^{\sigma t^{*}} \cos \pi z^{*}, \quad D \phi_{3}{ }^{*}=H_{1} e^{\sigma t^{*}} \cos \pi z^{*}, \quad \phi_{3}{ }^{*}=\left(\frac{H_{1}}{\pi}\right) e^{\sigma t^{*}} \sin \pi z^{*}
\end{aligned}
$$

where $A_{1}, B_{1}, C_{l}, D_{l}, E_{l}, F_{l}, G_{l}$, and $H_{1}$ are constants and $\sigma$ is the growth rate, in, general, a complex constant. Substituting Eq.(4.7) in Eqs (3.28)-(4.5) and dropping asterisks for convenience, we get the following equations

$$
\begin{aligned}
& \left\{\sigma+\left(1+N_{1}\right)\left(\pi^{2}+a^{2}\right)\right\}\left(\pi^{2}+a^{2}\right) A_{1}+a \sqrt{R}\left[\left(M_{1}-M_{4}\right) C_{1}-\left(1+M_{1}-M_{4}\right) B_{1}\right]+ \\
& +a \sqrt{S_{1}}\left[\left(M_{1}^{\prime}-M_{4}^{\prime}\right) E_{1}+\left(1-M_{1}^{\prime}+M_{4}^{\prime}\right) F_{1}\right]+a \sqrt{S_{2}}\left[\left(M_{1}^{\prime \prime}-M_{4}^{\prime \prime}\right) H_{l}+\left(1-M_{1}^{\prime \prime}+M_{4}^{\prime \prime}\right) G_{1}\right]+ \\
& -2 N_{l}\left(\pi^{2}+a^{2}\right) D_{l}+\frac{T_{\mathrm{A}}}{\left\{\sigma+\left(1+N_{1}\right)\left(\pi^{2}+a^{2}\right)\right\}} \pi^{2} A_{l}=0 \text {, } \\
& \left.-2 N_{l}\left(\pi^{2}+a^{2}\right) A_{1}+I^{\prime} \sigma+4 N_{1}+N_{3}\left(\pi^{2}+a^{2}\right)\right\} D_{1}=0, \\
& \left(1-M_{2}\right) a \sqrt{R} A_{1^{-}}\left(\pi^{2}+a^{2}+\mathrm{P}_{\mathrm{r}} \sigma\right) B_{1}+\left(\mathrm{P}_{\mathrm{r}} M_{2} \sigma\right) C_{1}-a \sqrt{R} N_{5} D_{1}=0, \\
& \left(1-M_{2}^{\prime}\right) a \sqrt{S_{1}} A_{l^{-}}\left(\pi^{2}+a^{2}+P S_{1} \sigma\right) \mathrm{F}_{1}+\left(P S_{1} M_{2}^{\prime} \sigma\right) E_{l}=0,
\end{aligned}
$$




$$
\begin{aligned}
& \left(1-M_{2}^{\prime \prime}\right) a \sqrt{S_{2}} A_{1}-\left(\pi^{2}+a^{2}+P S_{2} \sigma\right) G_{1}+\left(P S_{2} M_{2}^{\prime \prime} \sigma\right) H_{1}=0, \\
& -\pi B_{1}+\left(\pi^{2}+a^{2} M_{3}\right) C_{1}=0, \\
& -\pi^{2} F_{1}+\left(\pi^{2}+a^{2} M_{3}\right) E_{1}=0, \\
& -\pi^{2} G_{1}+\left(\pi^{2}+a^{2} M_{3}\right) H_{1}=0 .
\end{aligned}
$$

For the existence of non-trivial solutions of the above equations, the determinant of the coefficients of $A_{l}, B_{l}, C_{l}, D_{l}, E_{l}, F_{l}, G_{l}$, and $H_{l}$ in Eqs (4.3)-(4.10) must vanish. This determinant on simplification yields

$$
i T_{5} \sigma_{i}^{5}+T_{4} \sigma_{i}^{4}-i T_{3} \sigma_{i}^{3}-T_{2} \sigma_{i}^{2}+i T_{1} \sigma_{i}+T_{0}=0 .
$$

Here

$$
\begin{aligned}
& T_{5}=b L_{4}^{\prime} L_{3}^{\prime} L_{2}^{\prime} I_{l} \\
& T_{4}=b\left[b L_{4}^{\prime} L_{1}\left(L_{3}^{\prime}+L_{2}^{\prime}\right) I_{1}+\left\{\left(1+N_{1}\right) b I_{1}+4 N_{1}+N_{3}^{\prime} b\right\} L_{4}^{\prime} L_{3}^{\prime} L_{2}^{\prime}+L_{3}^{\prime} L_{2}^{\prime} L_{1}\right] \text {, } \\
& T_{3}=b^{3} L_{1}\left[L_{4}^{\prime}\left(L_{3}^{\prime}+L_{2}^{\prime}\right)\left(1+N_{1}\right)+\left(L_{4}^{\prime} L_{1}+L_{2}^{\prime}\right)\right] \mathrm{I}_{1}+b^{2}\left[L_{4}^{\prime} L_{1}\left(4 N_{1}+N_{3}^{\prime} b\right)\left(L_{3}^{\prime}+L_{2}^{\prime}\right)+\right. \\
& \left.+L_{3}^{\prime}\left\{\left(1+N_{1}\right) L_{2}^{\prime}+L_{1}\right\} I_{1}\right]+b\left[L_{3}^{\prime} L_{2}^{\prime}\left(4 N_{1}+N_{3}^{\prime} b\right)\right]+L_{4}^{\prime} L_{3}^{\prime} L_{2}^{\prime} \\
& \left\{\left(1+N_{1}\right)\left(4 N_{1}+N_{3}^{\prime} b\right)\left(b^{3}+\frac{T_{A}}{(1+N)^{2}}\right)-4 N_{1}^{2} b^{3}\right\}+\left[-L_{4}^{\prime} L_{3}^{\prime} L_{2}\left(1-M_{2}\right)\left(x R_{1}\right)+\right. \\
& +L_{2}^{\prime}\left(1-M_{2}^{\prime}\right)\left(x S_{1}\right)+\left\{L_{3}^{\prime}+L_{1}^{\prime}\left(L_{1}-1\right\} L_{4}^{\prime} L_{5}\left(x S_{1}\right)\left(x S_{2}\right)\right] I_{1} \text {, } \\
& T_{2}=b^{4}\left[\left(L_{4}^{\prime} L_{1}^{2}+L_{2}^{\prime} L_{1}\right)\left(1+N_{1}\right) I_{1}+b^{3}\left[\left(L_{4}^{\prime} L_{1}+L_{2}^{\prime}\right) L_{1}\left(4 N_{1}+N_{3}^{\prime} b\right)+L_{3}^{\prime} L_{1}\left(1+N_{1}\right) I_{1}\right]+\right. \\
& +b^{2}\left[L_{3}^{\prime} L_{1}\left(4 N_{1}+N_{3}^{\prime} b\right)\right]+b\left[\left\{L_{2}^{\prime} L_{3}\left(1-M_{2}^{\prime}\right)\left(x S_{1}\right)-\left(1-M_{2}\right)\left(L_{4}^{\prime} L_{1}+L_{3}^{\prime}\right) L_{2}\left(x R_{1}\right)\right\} I_{1}+\right. \\
& \left.+\left\{L_{5} L_{4} L_{1}^{2}-L_{4} L_{1}^{\prime}\left(1-M_{2}^{\prime \prime}\right)\left(L_{1}-1\right)\right\}\left(x S_{1}\right)\left(x S_{2}\right) I_{1}+L_{4}^{\prime} L_{3}^{\prime} L_{2}\left(2 N_{1} N_{5}^{\prime}\right)\left(x R_{1}\right)\right]+ \\
& +\left[L_{4}^{\prime} L_{1}\left(L_{2}^{\prime}+L_{3}^{\prime}\right)+L_{3}^{\prime} L_{2}^{\prime}\right]\left\{\left(1+N_{1}\right)\left(4 N_{1}+N_{3}^{\prime} b\right)\left(b^{3}+\frac{T_{A}}{(1+N)^{2}}\right)-4 N_{1}^{2} b^{3}\right\}+ \\
& \left\{L _ { 1 } L _ { 4 } L _ { 3 } ^ { \prime } \left[+\left(L_{5} L_{4} L_{1}^{\prime}\left(L_{1}-1\right)\right\}\left(4 N_{1}+N_{3}^{\prime} b\right)\left(x S_{1}\right)\left(x S_{2}\right)-L_{4}^{\prime} L_{3}^{\prime} L_{2}\left(1-M_{2}\right)\left(4 N_{1}+\right.\right.\right. \\
& \left.N_{3}^{\prime} b\right)\left(x R_{1}\right)+\left(1-M_{2}^{\prime}\right) L_{4}^{\prime} L_{3}\left\{L_{2}^{\prime}\left(4 N_{1}+N_{3}^{\prime} \mathrm{b}\right)+I_{1}\right\}\left(x S_{1}\right) \text {, } \\
& T_{1}=b^{4}\left[L_{1}^{2}\left(4 N_{1}+N_{3}^{\prime} b\right)\right]+b^{2}\left[\left\{\left(1-M_{2}^{\prime}\right) I_{1} L_{1} L_{3}-\left(1-M_{2}^{\prime \prime}\right) I_{1} L_{1} L_{4}\left(L_{1}-1\right)\right\}\left(x S_{1}\right)+\right. \\
& \left.+\left\{2 N_{1} N_{5}^{\prime}\left(L_{4}^{\prime} L_{1}+L_{1}^{\prime} L_{3}\right)-L_{1} L_{2}\left(1-M_{2}\right) I_{1}\right\}\left(x R_{1}\right)\right]+ \\
& +b\left[( L _ { 4 } ^ { \prime } L _ { 1 } ^ { 2 } + L _ { 2 } ^ { \prime } L _ { 1 } + \dot { L } _ { 3 } ^ { \prime } L _ { 1 } ) \left\{\left\{\left(1+N_{1}\right)\left(4 N_{1}+N_{3}^{\prime} b\right)\left(b^{3}+\frac{T_{A}}{(1+N)^{2}}\right)-4 N_{1}^{2} b^{3}\right\}+\right.\right. \\
& +\left\{L_{5} L_{4} L_{1}^{2}-\left(1-M_{2}^{\prime \prime}\right) L_{4} L_{1}^{\prime}\left(L_{1}-1\right)\right\}\left(4 N_{1}+N_{3}^{\prime} b\right)\left(x S_{1}\right)\left(x S_{2}\right)+\left(L_{2}^{\prime} L_{3}+L_{4}^{\prime} L_{1} L_{3}\right) \\
& \left.\left\{\left(1-M_{2}^{\prime}\right)\left(4 N_{1}+N_{3}^{\prime} b\right)\left(x S_{1}\right)\right\}-\left(1-M_{2}\right) L_{2} L_{1}\left(L_{4}^{\prime}+L_{3}^{\prime}\right)\left(4 N_{1}+N_{3}^{\prime} b\right)\left(x R_{1}\right)\right] \text {, } \\
& T_{0}=b^{3}\left[L_{1}\left(2 N_{1} N_{5}^{\prime}\right)\left(x R_{1}\right)+b^{2}\left\{\left[\left(1+N_{1}\right)\left(4 N_{1}+N_{3}^{\prime} b\right)\left(b^{3}+\frac{T_{A}}{(1+N)^{2}}\right)-4 N_{1}^{2} b^{3}\right]+\right.\right. \\
& \left.+\left[\left(1-M_{2}^{\prime}\right) L_{1} L_{3}\left(x S_{1}\right)-\left(1-M_{2}{ }^{\prime \prime}\right) L_{1} L_{4}\left(L_{1}-1\right)\right)\left(x S_{1}\right)\left(x S_{2}\right)\right\}\left(4 N_{1}+N_{3}^{\prime} b\right)+ \\
& \text { - } \left.\left.\left(1-M_{2}\right) L_{1} L_{2}\left(4 N_{1}+N_{3}^{\prime} b\right)\left(x R_{1}\right)\right]\right\}
\end{aligned}
$$

where

$$
R_{l}=\frac{R}{\pi^{4}}, \quad T_{A_{l}}=\frac{T_{A}}{\pi^{4}}, \quad S_{1}=\frac{S_{1}}{\pi^{4}}, \quad S_{2}=\frac{S_{2}}{\pi^{4}}, \quad x=\frac{a^{2}}{\pi^{2}}, \quad I_{l}=\pi^{2} I^{\prime}, \quad i \sigma_{i}=\frac{\sigma}{\pi^{2}},
$$




$$
\begin{aligned}
& N_{3}^{\prime}=\pi^{2} N_{3}, \quad N_{5}^{\prime}=\pi^{2} N_{5}, \quad b=1+x, \quad L_{1}=\left(1+x M_{3}\right), \quad L_{1}^{\prime}=P s_{1}\left(1+x M_{3}\right), \\
& L_{2}=\left[1+x M_{3}+x M_{3} M_{1}\left(1-M_{5}\right)\right], \quad L_{2}^{\prime}=\mathrm{P}_{\mathrm{r}}\left[\left(1-M_{2}\right)+x M_{3}\right], \\
& L_{3}=\left[1+x M_{3}^{\prime}+x M_{3} M_{1}^{\prime}\left(\frac{1}{M_{5}^{\prime}}-1\right)\right], \quad L_{3}^{\prime}=P s_{1}\left(1-M_{2}^{\prime}+x M_{3}\right), \\
& L_{4}=\left[1+x M_{3}+x M_{3} M_{1}^{\prime \prime}\left(\frac{1}{M_{5}^{\prime}}-1\right)\right], \quad L_{4}^{\prime}=P s_{2}\left(1-M_{2}^{\prime \prime}+x M_{3}\right), \quad L_{5}=\mathrm{P}_{\mathrm{r}}\left(1-M_{2}^{\prime \prime}\right) .
\end{aligned}
$$

\section{Results and discussion}

\subsection{The case of stationary convection}

When the instability sets in as stationary convection in the case $M_{2} \cong 0, M_{2}^{\prime} \cong 0$, the marginal state will be characterized by $\sigma_{i}=0$ (Chandrasekhar, 1981), then the Rayleigh number $\mathrm{R}_{1}$ is given by

$$
\begin{aligned}
& \left(1+x M_{3}\right)\left\{\left(1+N_{1}\right)\left(4 N_{1}+N_{3}^{\prime} b\right)\left[b^{3}+\frac{T_{A_{1}}}{\left(1+N_{1}\right)^{2}}\right]-4 N_{1}^{2} b^{3}\right\}+ \\
R_{1}= & +\left[\left\{1+x M_{3}+x M_{1}^{\prime} M_{3}\left(\frac{1}{M_{5}}-1\right)\right\}\left(x S_{1}\right)+\left\{1+x M_{3}+x M_{1}^{\prime \prime} M_{3}\left(\frac{1}{M_{5}}+1\right)\right\}\left(x S_{2}\right)\right]\left(4 N_{1}+N_{3}^{\prime} b\right) \\
X\left\{1+x M_{3}+x M_{1} M_{3}\left(1-M_{5}\right)\right\}\left\{4 N_{1}+b\left(N_{3}^{\prime}-2 N_{1} N_{5}^{\prime}\right)\right\} &
\end{aligned}
$$

which expresses the modified Rayleigh number $\mathrm{R}_{1}$ as a function of dimensionless wave number $x$, the buoyancy magnetization parameter $M_{1}$, the non-buoyancy magnetization parameter $M_{3}$, solute gradient parameters $S_{1}$ and $S_{2}$, ratio of the salinity effect on the magnetic field to the pyromagnetic coefficient $M_{5}$, coupling parameter $N_{l}$ (coupling between vorticity and spin effects), spin diffusion parameter $N_{3}$ and micropolar heat conduction parameter $N_{5}^{\prime}$ (coupling between spin and heat fluxes). The parameters $N_{l}$ and $N_{3}^{\prime}$ measure the micropolar viscous effect and micropolar diffusion effect, respectively.

The classical results in respect of Newtonian fluids can be obtained as the limiting case of the present study. Setting $N_{l}=0$ and $S_{l}=0$, and keeping $N_{3}{ }^{\prime}$ arbitrary in Eq.(5.1), we get

$$
R_{1}=\frac{\left(1+x M_{3}\right)\left\{(1+x)^{3}+T_{A_{1}}\right\}}{x\left\{1+x M_{3}\left(1+M_{1}\right)\right\}},
$$

which is the expression for the Rayleigh number of ferromagnetic fluids (Finlayson, 1975).

Setting $M_{3}=0$ in Eq.(5.2), we get

$$
R_{l}=\frac{\left\{(1+x)^{3}+T_{A_{1}}\right\}}{x},
$$


the classical Rayleigh Bénard result (Chandrasekhar, 1981), for the Newtonian fluid case.

Before we investigate the effects of various parameters, we first make some comments on the parameters $N_{l}, N_{3}{ }^{\prime}$ and $N_{5}{ }^{\prime}$ arising due to suspended particles. Assuming the Clausius- Duhem inequality, Eringen (1972) presented certain thermodynamic restrictions which lead to non-negativeness of $N_{1}, N_{3}^{\prime}$ and $N_{5}^{\prime}$. It is obvious that couple stress comes into play at small values of $N_{3}^{\prime}$. This supports the condition that $0 \leq N_{1} \leq 1$ and that $N_{3}{ }^{\prime}$ is a small positive real number. The parameter $N_{5}{ }^{\prime}$ has to be finite because the increasing of concentration has to be practically stopped somewhere and hence it has to be a positive, finite real number. The range of the values for the other parameters is as in the classical ferroconvection problem involving a Newtonian ferromagnetic fluid (Sharma and Kumar, 1994; Sastry and Rao, 1983; Bhattaharya and Abbas, 1985). $M_{1}^{\prime}$ 'and $M_{1}^{\prime \prime}$ is the effect of magnetization due to salinity. This is allowed to vary from 0.1 to 0.5 with values smaller than the magnetization parameter $M_{3} . M_{5}$ represents the ratio of the salinity effects on the magnetic field to the pyromagnetic coefficient. This is varied between 0.1 to 0.5 . The salinity Rayleigh numbers $S_{1}$ and $S_{2}$ vary from 0 to 500 .

To investigate the effect of solute gradients, the non-buoyancy magnetization coefficient, coupling parameter, spin parameter, and micropolar heat conduction parameter, we examine the behavior of $\frac{d R_{1}}{d T_{A}}, \frac{d R_{1}}{d S_{1}}, \frac{d R_{1}}{d S_{2}}, \frac{d R_{1}}{d M_{3}}, \frac{d R_{1}}{d N_{1}}, \frac{d R_{1}}{d N_{3}^{\prime}}$ and $\frac{d R_{1}}{d N_{5}^{\prime}}$ analytically. Equation (5.1) gives

$$
\frac{d R_{1}}{d T_{A}}=\frac{L_{1}\left(4 N_{1}+N_{3}^{\prime} b\right)}{x\left(1+N_{1}\right) L_{2}\left\{4 N_{1}+b\left(N_{3}^{\prime}-2 N_{1} N_{5}^{\prime}\right)\right\}},
$$

which is positive if

$$
N_{3}^{\prime}>2 N_{1} N_{5}^{\prime}
$$

which shows that rotation has a stabilizing effect when condition (5.4) holds. In the absence of the micropolar viscous effect $\left(N_{l}=0\right)$, rotation always has a stabilizing effect on the system.

$$
\begin{aligned}
& \frac{d R_{1}}{d S_{1}}=\frac{\left[1+x M_{3}+x M_{1}^{\prime} M_{3}\left(\frac{1}{M_{5}}-1\right)\right]\left(4 N_{1}+N_{3}^{\prime} b\right)}{L_{2}\left\{4 N_{1}+b\left(N_{3}^{\prime}-2 N_{1} N_{5}^{\prime}\right)\right\}}, \\
& \frac{d R_{1}}{d S_{2}}=\frac{\left[1+x M_{3}+x M_{1}^{\prime \prime} M_{3}\left(\frac{1}{M_{5}}-1\right)\right]\left(4 N_{1}+N_{3}^{\prime} b\right)}{L_{2}\left\{4 N_{1}+b\left(N_{3}^{\prime}-2 N_{1} N_{5}^{\prime}\right)\right\}},
\end{aligned}
$$

This shows that, for a stationary convection, the stable solute gradients have a stabilizing effect, if

$$
N_{3}^{\prime}>2 N_{1} N_{5}^{\prime}
$$

In the absence of the micropolar viscous effect (coupling parameter $N_{1}$ ), stable solute gradients always have a stabilizing effect on the system. Equation (5.1) also yields 


$$
\begin{gathered}
\left(1-M_{5}\right) b\left[b^{2} M_{1}\left\{\left(1+N_{1}\right) N_{3}^{\prime} b+4 N_{1}\right\}+\frac{\left(N_{3}^{\prime} b+4 N_{1}\right) T_{A_{1}}}{b\left(1+N_{1}\right)}\right]+ \\
\frac{d R_{1}}{d M_{3}}=\frac{+\left[\left(x S_{1}\right)\left(M_{1}-\frac{M_{1}^{\prime}\left(1+x M_{3}\right)}{M_{5}}\right)+\left(x S_{2}\right)\left(M_{1}-\frac{M_{1}^{\prime \prime}\left(1+x M_{3}\right)}{M_{5}}\right)\right]\left(4 N_{1}+N_{3}^{\prime} b\right)}{\left\{1+x M_{3}+x M_{1} M_{3}\left(1-M_{5}\right)\right\}^{2}\left\{4 N_{1}+b\left(N_{3}^{\prime}-2 N_{1} N_{5}^{\prime}\right)\right\}},
\end{gathered}
$$

which is negative, if

$$
N_{3}^{\prime}>2 N_{1} N_{5}^{\prime}, \quad M_{4}>\left(1+x M_{3}\right) \quad \text { and } \quad M_{4}>M_{1}^{\prime \prime}\left(1+x M_{3}\right)
$$

This shows that the non-buoyancy magnetization has a destabilizing effect when conditions (5.9) hold. In the absence of the micropolar viscous effect $\left(N_{I}=0\right)$ and the effect on magnetization due to salinity $\left(M_{l}^{\prime}=0\right.$ and $\left.M_{1}^{\prime \prime}=0\right)$, the non-buoyancy magnetization always has a destabilizing effect on the system.

It follows from Eq.(5.1) that

$$
\begin{aligned}
& b L_{1}\left[b^{4} N_{3}^{\prime}\left\{2 N_{5}^{\prime}+\frac{N_{3}^{\prime}}{\left(1+N_{1}\right)^{2}}\left[b\left(1+N_{1}\right)^{2}-\frac{T_{A_{l}}}{b^{2}}\right]\right\}+\right. \\
&+\frac{T_{A_{1}}}{b\left(1+N_{1}\right)^{2}}\left\{4 b N_{1} N_{3}^{\prime}+2\left(b N_{5}^{\prime}-2\right)\left\{4 N_{1}^{2}+b N_{3}^{\prime}+\left(2 N_{1}+1\right)\right\}\right\}+ \\
&+\left(2 b^{2} N_{3}^{\prime} N_{5}^{\prime}\right)\left[\left(x S_{1}\right)\left\{1+x M_{3}+x M_{1}^{\prime} M_{3}\left(\frac{1}{M_{5}}-1\right)\right\}+\right. \\
& \frac{d R_{1}}{d N_{1}}=\left.+\left(x S_{2}\right)\left\{1+x M_{3}+x M_{1}^{\prime \prime} M_{3}\left(\frac{1}{M_{5}}-1\right)\right\}\right] \\
& x L_{2}\left\{4 N_{1}+b\left(N_{3}^{\prime}-2 N_{1} N_{5}^{\prime}\right)\right\}^{2}
\end{aligned}
$$

which is positive if

$$
T_{A_{1}}<1 \quad \text { and } \quad N_{5}^{\prime}>2 .
$$

This shows that the coupling parameter always has a stabilizing effect when condition (5.11) holds. In the absence of rotation $\left(T_{A_{l}}=0\right)$, Eq.(5.11) yields that $\frac{d R_{1}}{d N_{l}}$ is always positive, implying thereby the stabilizing effect of the coupling parameter. Thus, the stabilizing behavior of the coupling parameter is virtually unaffected by magnetization parameters but it is significantly affected by the micropolar heat conduction $N_{5}{ }^{\prime}$ and by Taylor $T_{A_{1}}$.

Equation (5.1) gives the number 


$$
\frac{d R_{1}}{d N_{3}^{\prime}}=\frac{2 b^{4} L_{1} N_{l}\left[N_{5}^{\prime} b+N_{l}\left(b N_{5}^{\prime}-2\right)+\frac{T_{A_{1}} N_{5}^{\prime}}{b^{2}\left(1+N_{1}\right)}\right]}{x L_{2}\left\{4 N_{1}+b\left(N_{3}^{\prime}-2 N_{1} N_{5}^{\prime}\right)\right\}^{2}},
$$

which is negative if $N_{5}{ }^{\prime}>2$.

This shows that the spin diffusion has a stabilizing effect when condition (5.12) holds. Equation (5.1) also gives

$$
\begin{gathered}
2 b^{2} L_{1} N_{1}\left\{b^{2}\left[\left(1+N_{1}\right) N_{3}^{\prime} b+4 N_{1}\right]+\frac{\left(N_{3}^{\prime} b+4 N_{1}\right) T_{A_{l}}}{b\left(1+N_{l}\right)}\right\}+ \\
2 b\left(4 N_{1}+b N_{3}^{\prime}\right)+\left[\left(x S_{1}\right)\left\{1+x M_{3}+x M_{1}^{\prime} M_{3}\left(\frac{1}{M_{5}}-1\right)\right\}+\right. \\
\frac{d R_{1}}{d N_{5}^{\prime}}=\frac{\left.+\left(x S_{2}\right)\left\{1+x M_{3}+x M_{1}^{\prime \prime} M_{3}\left(\frac{1}{M_{5}}-1\right)\right\}\right]}{x L_{2}\left\{4 N_{1}+b\left(N_{3}^{\prime}-2 N_{1} N_{5}^{\prime}\right)\right\}^{2}},
\end{gathered}
$$

which is always positive.

This shows that the micropoalr heat conduction always has a stabilizing effect.

For sufficiently large values of $M_{l}$ (Finalyson, 1970), we obtain the results for the magnetic mechanism

$$
\begin{aligned}
& b^{2}\left(1+x M_{3}\right)\left\{\left(1+N_{1}\right)\left(4 N_{1}+N_{3}^{\prime} b\right)\left\{b+\frac{T_{A_{1}}}{b^{2}\left(1+N_{1}\right)^{2}}\right\}+4 N_{1}^{2}\right\}+ \\
&+\left(4 N_{1}+b N_{3}^{\prime}\right)\left[\left(x S_{1}\right)\left\{1+x M_{3}+x M_{1}^{\prime} M_{3}\left(\frac{1}{M_{5}}-1\right)\right\}+\right. \\
& \mathrm{R}_{\mathrm{m}}=R_{1} M_{1}=\left.+\left(x S_{2}\right)\left\{1+x M_{3}+x M_{1}^{\prime \prime} M_{3}\left(\frac{1}{M_{5}}-1\right)\right\}\right] \\
& x^{2} M_{3}\left(1+M_{5}\right)\left\{4 N_{1}+b\left(N_{3}^{\prime}-2 N_{1} N_{5}^{\prime}\right)\right\}
\end{aligned}
$$

where $\mathrm{R}_{\mathrm{m}}$ is the magnetic thermal Rayleigh number.

As a function of $x, \mathrm{R}_{\mathrm{m}}$ given by Eq.(5.16) attains its maximum when

$$
P_{6} x^{6}+P_{5} x^{5}+P_{4} x^{4}+P_{3} x^{3}+P_{2} x^{2}+P_{1} x+P_{0}=0 .
$$

The coefficients $P_{0}, P_{1}, P_{2}, P_{3}, P_{4}, P_{5}, P_{6}$ being quite lengthy, have not been written here and are evaluated on numerical calculations.

The values of the critical wave number for the onset of instability are determined numerically using the Newtonian Raphson method by the condition $\frac{d R_{m}}{d x}=0$. With $x_{1}$ determined as a solution of Eq.(5.15), Eq.(5.14) will give the required critical magnetic thermal Rayleigh number $N_{c}$ which depends upon $M_{3}, S_{1}, S_{2}$ and micropolar parameters $N_{1}, N_{3}$ and $N_{5}$. 


\subsection{Principle of exchange of stabilities}

Here we examine the possibility of oscillatory modes, if any, on the stability problem due to the presence of micropolar parameters and solute gradients. Equating the imaginary parts of Eq.(4.11), we obtain

$$
\begin{aligned}
& \sigma_{i}\left[\left\{b L_{4}^{\prime} L_{3}^{\prime} L_{2}^{\prime} I_{1}\right\} \sigma_{i}^{4}-\left\{b ^ { 4 } \left[\left(L_{4}^{\prime} L_{1}^{2}+L_{2}^{\prime} L_{1}\right)\left(1+N_{1}\right) I_{1}+b^{3}\left[\left(L_{4}^{\prime} L_{1}+L_{2}^{\prime}\right) L_{1}\left(4 N_{1}+N_{3}^{\prime} b\right)+L_{3}^{\prime} L_{1}\left(1+N_{1}\right) I_{1}\right]+\right.\right.\right. \\
& +b^{2}\left[L_{3}^{\prime} L_{1}\left(4 N_{1}+N_{3}^{\prime} b\right)\right]+b\left[\left\{L_{2}^{\prime} L_{3}\left(1-M_{2}^{\prime}\right)\left(x S_{1}\right)-\left(1-M_{2}\right)\left(L_{4}^{\prime} L_{1}+L_{3}^{\prime}\right) L_{2}\left(x R_{1}\right)\right\} I_{1}+\right. \\
& \left.+\left\{L_{5} L_{4} L_{1}^{2}-L_{4} L_{1}^{\prime}\left(1-M_{2}^{\prime \prime}\right)\left(L_{1}-1\right)\right\}\left(x S_{1}\right)\left(x S_{2}\right) I_{1}+L_{4}^{\prime} L_{3}^{\prime} L_{2}\left(2 N_{1} N_{5}^{\prime}\right)\left(x R_{1}\right)\right]+ \\
& +\left[L_{4}^{\prime} L_{1}\left(L_{2}^{\prime}+L_{3}^{\prime}\right)+L_{3}^{\prime} L_{2}^{\prime}\right]\left\{\left(1+N_{1}\right)\left(4 N_{1}+N_{3}^{\prime} b\right)\left(b^{3}+\frac{T_{A}}{(1+N)^{2}}\right)-4 N_{1}^{2} b^{3}\right\}+\left[\left\{L_{1} L_{4} L_{3}^{\prime}+\right.\right. \\
& +\left(L_{5} L_{4} L_{1}^{\prime}\left(L_{1}-1\right)\right\}\left(4 N_{1}+N_{3}^{\prime} b\right)\left(x S_{1}\right)\left(x S_{2}\right)-L_{4}^{\prime} L_{3}^{\prime} L_{2}\left(1+M_{2}\right)\left(4 N_{1}+N_{3}^{\prime} b\right)\left(x R_{1}\right)+ \\
& \left.+\left(1-M_{2}^{\prime}\right) L_{4}^{\prime} L_{3}\left\{L_{2}^{\prime}\left(4 N_{1}+N_{3}^{\prime} b\right)+I_{1}\right\}\left(x S_{1}\right)\right\} \sigma_{i}^{2}\left\{b^{4}\left(4 N_{1}+N_{3}^{\prime} b\right)+\right. \\
& +b^{2}\left[\left\{\left(1-M_{2}^{\prime}\right) I_{1} L_{1} L_{3}-\left(1-M_{2}^{\prime \prime}\right) I_{1} L_{1} L_{4}\left(L_{1}-1\right)\right\}\left(x S_{1}\right)+\left\{2 N_{1} N_{5}^{\prime}\left(L_{4}^{\prime} L_{1}+L_{1}^{\prime} L_{3}\right)-L_{1} L_{2}\left(1+M_{2}\right) I_{1}\right\}\left(x R_{1}\right)\right]+ \\
& +b\left[( L _ { 4 } ^ { \prime } L _ { 1 } ^ { 2 } + L _ { 2 } ^ { \prime } L _ { 1 } + L _ { 3 } ^ { \prime } L _ { 1 } ) \left\{\left\{\left(1+N_{1}\right)\left(4 N_{1}+N_{3}^{\prime} b\right)\left(b^{3}+\frac{T_{A}}{(1+N)^{2}}\right)-4 N_{1}^{2} b^{3}\right\}+\right.\right. \\
& +\left\{L_{5} L_{4} L_{1}^{2}-\left(1-M_{2}^{\prime \prime}\right) L_{4} L_{1}^{\prime}\left(L_{1}-1\right)\right\}\left(4 N_{1}+N_{3}^{\prime} b\right)\left(x S_{1}\right)\left(x S_{2}\right)+\left(L_{2}^{\prime} L_{3}+L_{4}^{\prime} L_{1} L_{3}\right) \\
& \left.\left\{\left(1-M_{2}^{\prime}\right)\left(4 N_{1}+N_{3}^{\prime} b\right)\left(x S_{1}\right)\right\}-\left(1-M_{2}\right) L_{2} L_{1}\left(L_{4}^{\prime}+L_{3}^{\prime}\right)\left(4 N_{1}+N_{3}^{\prime} b\right)\left(x R_{1}\right)\right\}=0 .
\end{aligned}
$$

It is evident from Eq.(5.16) that $\sigma_{i}$ may be either zero or non-zero, meaning that the modes may be either oscillatory or oscillatory. In the absence of the micropolar viscous effect $\left(N_{I}=0\right)$, microinertia $\left(I_{I}=0\right)$ and solute gradients $\left(S_{1}=0, S_{2}=0, L_{3}^{\prime}=0\right.$, and $\left.L_{4}^{\prime}=0\right)$, we obtain the result as

$$
\sigma_{i}\left[L_{1}+L_{2}^{\prime}\right]=0 \text {. }
$$

Here the quantity inside the bracket is positive definite because the typical values of $M_{2}$ are $+10^{-6}$ (Finlayson, 1970). Hence

$$
\sigma_{i}=0
$$

which implies that the oscillatory modes are not allowed and the principle of exchange of stabilities is satisfied for a micropolar ferromagnetic fluid heated from below, in the absence of the micropolar viscous effect, microinertia and solute gradients. Thus from Eq.(5.17), we conclude that the oscillatory modes are introduced due to the presence of the micropolar viscous effect, microinertia and solute gradient, which are non-existent in their absence. Thus, it is important to note that the Taylor number $T_{A_{1}}$, gives a significant contribution to the development of oscillatory modes in the stability analysis. 


\subsection{The case of overstability}

The present section is devoted to finding whether the observed instability may really be overstability. Since we wish to determine the Rayleigh number for the onset of instability through the state of pure oscillations, it suffices to find conditions for which (4.11) will admit solutions with $\sigma_{i}$ real.

Equating real and imaginary parts of Eq.(4.11) and eliminating $R_{l}$ between them, we obtain

$$
A_{3} c_{1}^{3}+A_{2} c_{1}^{2}+A_{1} c_{1}+A_{0}=0
$$

where, $c_{l}=\sigma_{i}^{2}$, Since $\sigma_{i}$ is real for overstability, the three values of $c_{l}\left(=\sigma_{i}^{2}\right)$ are positive. The product of roots of Eq.(5.19) is $-\frac{A_{0}}{A_{3}}$, where

$$
\begin{aligned}
& A_{3}=-b L_{4}^{\prime 2} L_{3}^{\prime 2} L_{2}^{\prime} I_{1}\left\{\left[L_{1}+\left(1+N_{1}\right) L_{2}^{\prime}\right]\left(1-M_{2}\right)+\left(2 N_{1} N_{3}^{\prime}\right) L_{2}^{\prime}\right\} \\
& A_{0}=\left\{b^{3} L_{1}\left(2 N_{1} N_{5}^{\prime}\right)-b^{2}\left(1-M_{2}\right) L_{1} L_{2}\left(4 N_{1}+N_{3}^{\prime} b\right)\right\}\left\{b^{4} L_{1}^{2}\left(4 N_{1}+N_{3}^{\prime} b\right)+b^{2}\left[\left(1-M_{2}^{\prime}\right) I_{1} L_{1} L_{3}-\left(1-M_{2}^{\prime \prime}\right) I_{1}\right.\right. \\
& \left.L_{1} L_{4}\left(L_{1}-1\right)\right\}\left(x S_{1}\right)+b\left\{\left[\left(L_{4}^{\prime} L_{1}^{2}+L_{2}^{\prime} L_{1}+L_{3}^{\prime}\right)\left\{\left(1+N_{1}\right)\left(4 N_{1}+N_{3}^{\prime} b\right)\left(b^{3}+\frac{T_{A}}{(1+N)^{2}}\right)-4 N_{1}^{2} b^{3}\right\}\right]+\right. \\
& \left\{L_{5} L_{4} L_{1}^{2}-\left(1-M_{2}^{\prime \prime}\right) L_{4} L_{1}^{\prime}\left(L_{1}-1\right)\right\}\left(4 N_{1}+N_{3}^{\prime} b\right)\left(x S_{1}\right)\left(x S_{2}\right) I_{1}+\left(L_{2}^{\prime} L_{3}+L_{4}^{\prime} L_{1} L_{3}\right) \\
& \left.\left.\left\{\left(1-M_{2}^{\prime}\right)\left(4 N_{1}+N_{3}^{\prime} b\right)\left(x S_{1}\right)\right\}\right]\right\}-b_{2}\left\{\left[L_{1}^{2}\left\{\left(1-N_{1}\right)\left(4 N_{1}+N_{3}^{\prime} b\right)\left(b^{3}+\frac{T_{A}}{(1+N)^{2}}\right)-4 N_{1}^{2} b^{3}\right\}\right]+\right. \\
& \left.+\left[\left\{\left(1-M_{2}^{\prime}\right) L_{1} L_{3}\left(x S_{1}\right)-\left(1-M_{2}^{\prime \prime}\right) L_{1} L_{4}\left(L_{1}-1\right)\left(x S_{1}\right)\left(x S_{2}\right)\right\}\left(4 N_{1}+N_{3}^{\prime} b\right)\right]\right\} \\
& \left\{b^{2}\left[2 N_{1} N_{5}^{\prime}\left(L_{4}^{\prime} L_{1}+L_{1}^{\prime} L_{3}\right)-L_{1} L_{2}\left(1-M_{2}\right) I_{1}\right]\left(x R_{1}\right)-b\left(1-M_{2}\right) L_{2} L_{1}\left(L_{4}^{\prime}+L_{3}^{\prime}\right)\left(4 N_{1}+N_{3}^{\prime} b\right)\left(x R_{1}\right)\right\}
\end{aligned}
$$

The coefficients $A_{2}$ and $A_{1}$ being quite lengthy and not needed in the discussion of overstability, have not been written here.

Since $\sigma_{i}$ is real for overstability, the three values of $c_{l}\left(=\sigma_{i}^{2}\right)$ are positive. The product of roots of Eq.(5.19) is $-\frac{A_{0}}{A_{3}}$, and if this is to be negative, then $A_{3}$ and $A_{0}$ are of the same sign. Now, the product is negative if

$$
N_{3}^{\prime}\left(1-M_{2}\right)>4 N_{1} N_{5}^{\prime}, \quad N_{1}\left(1-M_{2}\right)>T_{A_{1}} I_{1} N_{5}^{\prime}, \quad N_{3}^{\prime} L_{2}^{\prime}>I_{1} L_{1}, \quad L_{1}>N_{1} L_{2}^{\prime}, \quad L_{2}^{\prime}>L_{1},
$$

and

$$
L_{2}^{\prime}>L_{3}^{\prime}, \quad\left(1+\frac{N_{3}^{\prime} L_{2}^{\prime}}{I_{1} L_{1}}\right), \quad L_{2}^{\prime}>L_{4}^{\prime}\left(1+\frac{N_{3}^{\prime} L_{2}^{\prime}}{I_{1} L_{1}}\right)
$$

i.e., if

$$
N_{3}^{\prime}>\frac{4 N_{1} N_{5}^{\prime}}{\left(1-M_{2}\right)}, \quad T_{A_{1}}<\frac{N_{1}\left(1-M_{2}\right)}{I_{1} N_{5}^{\prime}}, \quad N_{3}^{\prime}>\frac{I_{1}\left(1+x M_{3}\right)}{\operatorname{Pr}\left(1-M_{2}\right)},
$$




$$
\frac{1}{\operatorname{Pr}}>N_{1}, \quad \operatorname{Pr}>\frac{\left(1+x M_{3}\right)}{\left(1-M_{2}\right)}, \quad \operatorname{Pr}>P_{S_{1}}\left(1+\frac{\eta^{\prime} \operatorname{Pr}}{\eta I}\right),
$$

and

$$
\operatorname{Pr}>P S_{1}\left(1+\frac{\eta^{\prime} \operatorname{Pr}}{\eta I}\right)+\operatorname{Pr} M_{2}, \quad \operatorname{Pr}>P_{S_{2}}\left(1+\frac{\eta^{\prime} \operatorname{Pr}}{\eta I}\right) \quad \text { and } \quad \operatorname{Pr}>P_{S_{2}}\left(1+\frac{\eta^{\prime} \operatorname{Pr}}{\eta I}\right)+\operatorname{Pr} M_{2} P_{r}
$$

which implies that

$$
\begin{aligned}
& N_{3}^{\prime}>\max \left\{\frac{4 N_{1} N_{5}^{\prime}}{\left(1-M_{2}\right)}, \frac{I_{1}\left(1+x M_{3}\right)}{\operatorname{Pr}\left(1-M_{2}\right)}\right\}, \quad \frac{1}{N_{1}}>\operatorname{Pr}>\frac{\left(1+x M_{3}\right)}{\left(1-M_{2}\right)}, \quad K_{1}<K_{1}^{\prime}\left[\frac{\rho C_{1} \frac{\mu_{0} T_{0} K_{2}^{2}}{(1+\chi)}}{\rho C_{1}^{\prime}\left(1+\frac{\eta^{\prime} \operatorname{Pr}}{\eta I}\right)}\right], \\
& K_{1}<K_{l}^{\prime \prime}\left[\frac{\rho C_{1}-\frac{\mu_{0} T_{0} K_{2}^{2}}{(1+\chi)}}{\rho C_{1}^{\prime}\left(1+\frac{\eta^{\prime} \operatorname{Pr}}{\eta I}\right)}\right], \quad T_{A_{1}}=\frac{N_{1}\left(1-M_{2}\right)}{I_{1} N_{5}^{\prime}} .
\end{aligned}
$$

However $\mathrm{P}_{\mathrm{r}}>P_{S_{1}}\left(1+\frac{\eta^{\prime} \mathrm{P}_{\mathrm{r}}}{\eta I}\right)$ and $\mathrm{P}_{\mathrm{r}}>P_{S_{2}}\left(1+\frac{\eta^{\prime} \mathrm{P}_{\mathrm{r}}}{\eta I}\right)$ are already satisfied in the above condition because the typical values of $M_{2}$ are $+10^{-6}$ (Finalyson, 1970).

Thus, for $N_{3}^{\prime}>\max \left\{\frac{4 N_{1} N_{5}^{\prime}}{\left(1-M_{2}\right)}, \frac{I_{1}\left(1+\mathrm{x} M_{3}\right)}{P_{r}\left(1-M_{2}\right)}\right\}, \frac{1}{N_{1}}>\mathrm{P}_{\mathrm{r}}>\frac{\left(1+\mathrm{x} M_{3}\right)}{\left(1-M_{2}\right)}, \quad T_{A_{1}}<\frac{N_{1}\left(1-M_{2}\right)}{I_{1} N_{5}^{\prime}}$,

$$
K_{l}<K_{l}^{\prime}\left[\frac{\rho C_{1}-\frac{\mu_{0} T_{0} K_{2}^{2}}{(1+\chi)}}{\rho C_{I}^{\prime}\left(1+\frac{\eta^{\prime} P_{r}}{\eta I}\right)}\right] \text { and } K_{1}<K_{l}^{\prime \prime}\left[\frac{\rho C_{1}-\frac{\mu_{0} T_{0} K_{2}^{2}}{(1+\chi)}}{\rho C_{I}^{\prime}\left(1+\frac{\eta^{\prime} P_{r}}{\eta I}\right)}\right] \text {, }
$$

overstability cannot occur and the principle of the exchange of stabilities is valid. Hence the above conditions are the sufficient conditions for the non existence of overstability, the violation of which does not necessarily imply the occurrence of overstability. Rotation contributes two more conditions, i.e.

$$
\mathrm{P}_{\mathrm{r}}>\frac{\left(1+x M_{3}\right)}{\left(1-M_{2}\right)}, \quad \mathrm{T}_{A_{1}}<\frac{N_{1}\left(1-M_{2}\right)}{I_{1} N_{5}},
$$

for the non-existence of overstability. In rotating a non-magnetic fluid and in the absence of microrotation, the above condition reduces to $\mathrm{P}_{\mathrm{r}}>1$, which is in good agreement with the result obtained earlier (Chandrasekhar, 1981).

\section{Conclusions}

In this paper, the effect of rotation on triple - diffusive convection in a micropolar ferrofluid layer heated and soluted from below subjected to a transverse uniform magnetic field has been investigated. The behavior of various parameters like rotation parameter, solute gradients, non-buoyancy magnetization, 
coupling parameter, spin diffusive parameter and micropolar heat conduction on the onset of convection has been analyzed analytically and numerically. The results show that for the state of stationary convection, the non-buoyancy magnetization, spin diffusive parameter have a destabilizing effect under certain condition(s), whereas rotation, the coupling parameter and solute gradients have a stabilizing effect under certain condition(s). However, the micropolar heat conduction always has a stabilizing effect. The principle of exchange of stabilities is found to hold true for the micropolar ferrofluid heated from below in the absence of the micropolar viscous effect, microinertia, rotation and solute gradient. Thus oscillatory modes are introduced due to the presence of the micropolar viscous effect, microinertia, rotation and solute gradients, which were non-existent in their absence. In addition, the presence of rotation, solute gradients, coupling between vorticity and spin effect (micropolar viscous effect) and microinertia may bring overstability in the system. Finally, we conclude that rotation and micropolar parameters have a profound influence on triplediffusive convection in a micropolar ferrofluid layer heated and soluted from below.

\section{Acknowledgement}

I thank Dr. Sunil from Department of Applied Sciences, National Institute of Technology (Deemed University), Hamirpur, HP-177005, India for his valuable help in the completion of this paper.

\section{Nomenclature}

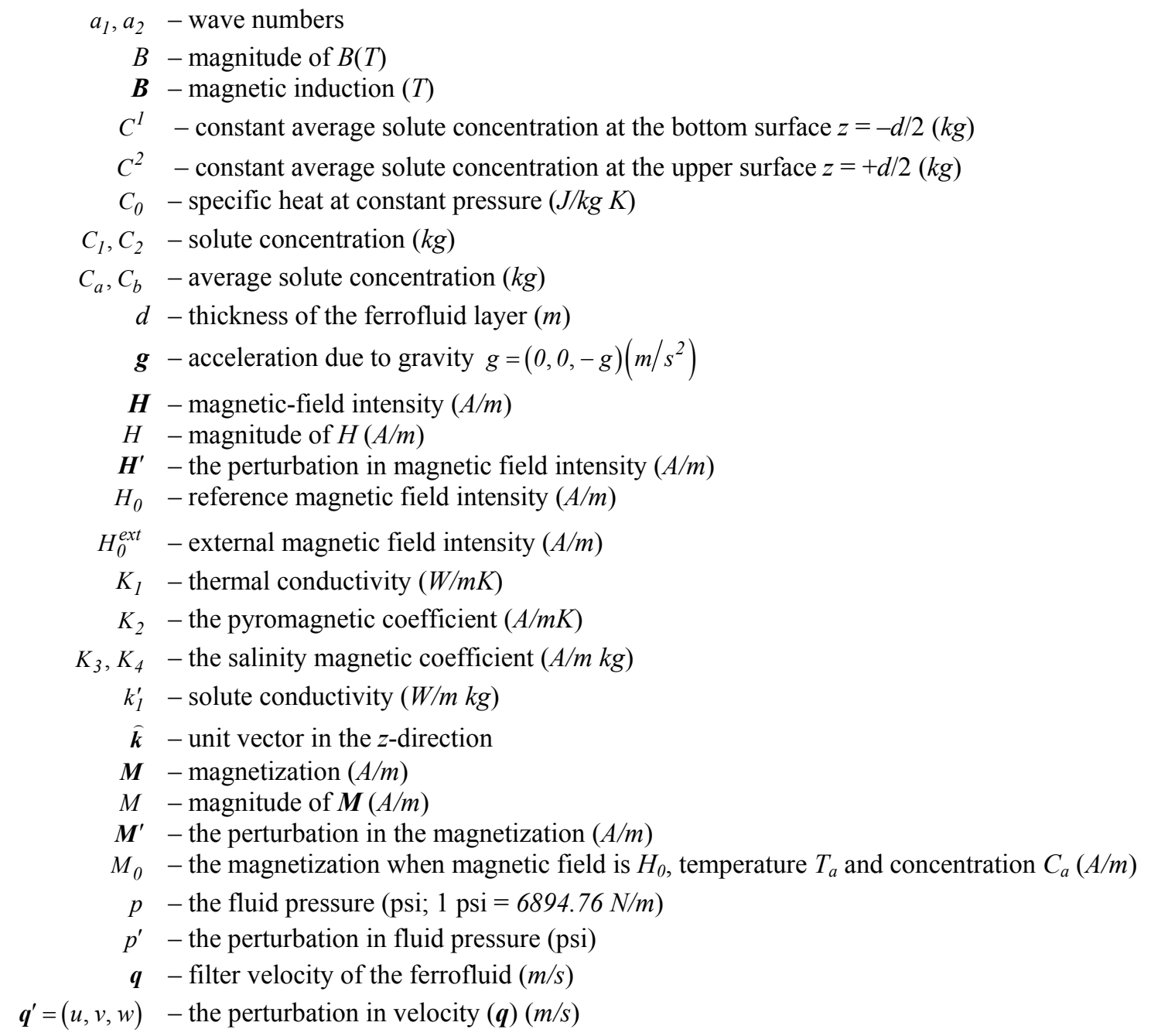


$T$ - temperature $(K)$

$T_{0}$ - constant average temperature at the bottom surface $z=-d / 2(K)$

$T_{1}$ - constant average temperature at the upper surface $z=+d / 2(K)$

$T_{a}$ - average temperature $(K)$

$t$ - time $(s)$

\section{Subscripts}

$$
\begin{array}{ll}
a & \text { - average } \\
b & \text { - basic state }
\end{array}
$$

$f$ - fluid

$\alpha-$ coefficient of thermal expansion $\left(K^{-1}\right)$

$\alpha^{\prime}, \alpha^{\prime \prime}$ - coefficient of solute expansion $\left(\mathrm{kg}^{-1}\right)$

$\beta$ - a uniform temperature gradient $(\mathrm{K} / \mathrm{m})$

$\beta^{\prime}, \beta^{\prime \prime}-$ a uniform solute gradient $(\mathrm{kg} / \mathrm{m})$

$\gamma, \gamma^{\prime}$ - the perturbation in concentration $C(\mathrm{~kg})$

$\delta$ - coefficient of MFD viscosity $\left(T^{-1}\right)$

$\varepsilon-$ porosity $\left(\mathrm{m}^{3} / \mathrm{m}^{3}\right)$

$\mu_{1} \quad$ - variable dynamic viscosity of fluid $(\mathrm{kg} /(\mathrm{m} \mathrm{s}))$

$\mu \quad$ - reference dynamic viscosity of fluid $(\mathrm{kg} /(\mathrm{m} \mathrm{s}))$

$\mu_{0}-$ magnetic permeability of free space $(\mathrm{H} / \mathrm{m})$

$\kappa-$ thermal diffusivity $\left(\mathrm{m}^{2} / \mathrm{s}\right)$

$\kappa^{\prime}, \kappa^{\prime \prime} \quad$ - solute diffusivity $\left(\mathrm{m}^{2} / \mathrm{s}\right)$

$v$ - kinematic viscosity $\left(\mathrm{m}^{2} / \mathrm{s}\right)$

$\rho$ - fluid density $\left(\mathrm{kg} / \mathrm{m}^{3}\right)$

$\rho^{\prime} \quad$ - perturbation in density $\rho\left(\mathrm{kg} / \mathrm{m}^{3}\right)$

$\rho_{0} \quad$ - reference density $\left(\mathrm{kg} / \mathrm{m}^{3}\right)$

$\vartheta \quad$ - perturbation in temperature $T(K)$

$\phi_{1}^{\prime}, \phi_{2}^{\prime}, \phi_{3}^{\prime} \quad$ - the perturbed magnetic potential $(A)$

$\chi$ - the magnetic susceptibility

$\nabla \quad$-gradient operator $\left(m^{-l}\right)$

\section{Non-dimensional parameters}

$M_{1}$ - buoyancy magnetization parameter

$M_{1}^{\prime}, M_{1}^{\prime \prime}$ - effect of magnetization due to salinity

$M_{3}$ - magnetic parameter (measures the linearity in the magnetic equation of state)

$M_{4}, M_{4}^{\prime}, M_{4}^{\prime \prime}$ - effect of magnetization due to solutes

$M_{5}$ - ratio of salinity effect on magnetic field to pyromagnetic coefficient

$N_{1}^{\prime}, N_{3}^{\prime}, N_{5}^{\prime}-$ micropolar parameters

Pr - Prandtl number

$S_{1}, S_{2}$ - solute gradient parameters

$x_{1}$ - dimensionless wave number

\section{References}

Abraham A. (2002): Rayleigh-Bénard convection in a micropolar ferromagnetic fluid. - Int. J. Eng. Sci., vol.40, No.4, pp.449-460.

Ahmadi A. (1976): Stability of a micropolar fluid layer heated from below. - Int. J. Eng. Sci., vol.14, No.1, pp.81-89. 
Bhattacharyya S.P. and Abbas M. (1985): On the stability of a hot rotating layer of micropolar fluid. - Int. J. Eng. Sci., vol.23, pp.371-374.

Bhattacharyya S.P. and Jena S.K. (1983): On the stability of a hot layer of micropolar fluid. - Int. J. Eng. Sci., vol.21, No.9, pp.1019-1024.

Chandrasekhar S. (1981): Hydrodynamic and Hydromagnetic Stability. - New York: Dover Publication.

Datta A.B. and Sastry V.U.K. (1976): Thermal instability of a horizontal layer of micropolar fluid heated from below. Int. J. Eng. Sci., vol.14, pp.631-637.

Eringen A.C.( 1966): Theory of micropolar fluids. - J. Math. Mech., vol.16, pp.1-18.

Eringen A.C. (1972): Theory of thermomicrofluids. - J. Math. Anal. Appl., vol.38, pp.480-496.

Finlayson B.A. (1970): Convective instability of ferromagnetic fluids. - J. Fluid Mech., vol.40, No.4, pp.753-767.

Gotoh K. and Yamada M. (1982): Thermal convection in a horizontal layer of magnetic fluids. - J. Phys. Soc. Jpn., vol.51, pp.3042-3048.

Kazakia Y. and Ariman T. (1971): Heat-conducting micropolar fluids. - Rheol. Acta., vol.10, pp.319-325.

Lalas D.P. and Carmi S. (1971): Thermoconvective stability of ferrofluids. - Phys. Fluids, vol.14, No.2, pp.436-437.

Lebon G. and Perez-Garcia C. (1981): Convective instability of a micropolar fluid layer by the method of energy. - Int. J. Eng. Sci., vol.19, No.10, pp.1321-1329.

Lopez Amalia R., Louis A. Romero and Pearlstein Arne J. (1990): Effect of rigid boundaries on the onset of convective instability in a triply diffusive fluid layer. - Phys. Fluids A, vol.2, pp.897.

Payne L.E. and Straughan B. (1989): Critical Rayleigh numbers for oscillatory and nonlinear convection in an isotropic thermomicropolar fluid. - Int. J. Eng. Sci., vol.27, No.7, pp.827-836.

Pearlstein A.J., Harris R.M. and Terrones G. (1989): The onset of convective instability in a triply diffusive fluid layer. - Journal of Fluid Mechanics, vol.202, pp.443-465.

Qin Y. and Kaloni P.N. (1992): A thermal instability problem in a rotating micropolar fluid. - Int. J. Eng. Sci., vol.30, pp.1117-1126.

Rosensweig R.E. (1995): Ferrohydrodynamics. - Cambridge: Cambridge University Press.

Sastry V.U.K. and Rammohan Rao V. (1983): Numerical solution of thermal instability of a rotating micropolar fluid layer. - Int. J. Eng. Sci., vol.21, pp.449.

Sharma R.C. and Kumar P. (1994): Effect of rotation on thermal convection in micropolar fluids. - Int. J. Eng. Sci., vol.32, pp.545-551.

Sharma R.C. and Kumar P. (1995): On micropolar fluids heated from below in hydromagnetics. - J. Non-Equilib. Thermodyn., vol.20, pp.150-159.

Sharma R.C. and Kumar P. (1997): On micropolar fluids heated from below in hydromagnetics in porous medium. Czech. J. Phys., vol.47, pp.637-647.

Sharma R.C. and Gupta U. (1995): Thermal convection in micropolar fluids in porous medium. - Int. J. Eng. Sci., vol..33, No.13, pp.1887-1892.

Siddheshwar P.G. (1993): Rayleigh-Be'nard convection in a ferromagnetic fluid with second sound. - Jpn. Soc. Mag. Fluids, vol.25, pp.32-36.

Siddheshwar P.G. (1995): Convective instability of ferromagnetic fluids bounded by fluid permeable magnetic boundaries. - J. Magn. Magn. Mater., vol.149 (1-2), pp.148-150.

Stiles P.J. and Kagan M. (1990): Thermoconvective instability of a horizontal layer of ferrofluid in a strong vertical magnetic field. - J. Magn. Magn. Mater., vol.85 (1-3), pp.196-198.

Straughan B. and. Walker D.W. (1997): Multi component diffusion and penetrative convection. - Fluid Dynamics Research, vol.19, pp.77-89. 
Sunil, Divya and Sharma R.C. (2004): Effect of rotation on ferromagnetic fluid heated and soluted from below saturating a porous Medium. - J. Geophys. Eng., vol.1, No.2, pp.116-127.

Sunil, Divya and Sharma V. (2005): Effect of dust particles on rotating ferromagnetic fluid heated from below saturating a porous Medium. - J. Colloid Interface Sci., vol.291, pp.152-161.

Sunil, Divya and Sharma R.C. ( 2005): Effect of dust particles on thermal convection in ferromagnetic fluid saturating a porous Medium. - J. Magn. Magn. Mater., vol.288, No.1, pp.183-195.

Sunil, Divya and Sharma R.C. (2005): The effect of magnetic field dependent viscosity on thermosolutal convection in ferromagnetic fluids aturating a porous medium. - Trans. Porous Media, vol.60, No.3, pp.251-274.

Sunil, Anu Sharma and Sharma R.C. (2006): Effect of dust particles on ferrofluid heated and soluted from below. - Int. J. Therm. Sci., vol.45, No.4, pp.347-358.

Sunil and Amit Mahajan (2009): A nonlinear stability analysis in a double- diffusive magnetized ferrofluid with magnetic -field dependent viscosity saturating a porous medium. - Can. J. Phys., vol.87, pp.659-673.

Sunil, Prakash C., Pavan K. and Mahajan A. (2008): Thermal convection in micropolar ferrofluid in the presence of rotation. - Journal of Magnetism and Magnetic Materials, vol.32, pp.316-324.

Zahn M. and Greer D.R. (1995): Ferrohydrodynamic pumping in spatially uniform sinusoidally time-varying magnetic fields. - J. Magn. Magn. Mater., vol.149 (1-2), pp.165-173.

Received: September 26, 2011

Revised: July 25, 2012 